\title{
Folic acid improves renal function — in what mechanism?
}

\author{
Kwas foliowy poprawia czynność nerek — w jakim mechanizmie?
}

\author{
Tomasz Stompór ${ }^{1}$, Anetta Undas ${ }^{2}$ \\ 'Department of Nephrology, Hypertension, and Internal Medicine, University of Warmia and Mazury, Olsztyn, Poland \\ ${ }^{2}$ Institute of Cardiology, Jagiellonian University Medical College, Krakow, Poland
}

In their interesting study published recently in "Kardiologia Polska" Mierzecki et al. [1] aimed to evaluate the effect of a diet containing low vs. moderate amounts of folic acid (FA) on several parameters of inflammation and blood coagulation, lipid profile, and renal function in apparently healthy young patients with risk factors for atherosclerosis. Additional administration of $0.4 \mathrm{mg} / \mathrm{d}$ FA reduced plasma concentrations of total homocysteine (tHcy) by about $20 \%$, in line with increased serum FA levels, regardless of the self-reported dietary intake of FA [1]. A higher intake of FA did not have a significant impact on any of the variables measured except for a $22.5 \%$ decrease in serum creatinine and $9.9 \%$ increase in estimated glomerular filtration rate (eGFR) in young subjects who reported FA intake below $40 \%$ of the reference value. To reinforce the concept of FA supplementation as a factor improving renal function, it would be of interest to assess associations between FA-induced changes in renal function and alterations in tHcy and FA concentrations. It is known that FA supplementation improves endothelial function via multiple mechanisms, and its noninvasive assessment (e.g. using tonometry) could validate this hypothesis [2], possibly confirming that this mechanism is important in increasing GFR observed in young people following FA. Moreover, given the key role of the kidneys in clearance of Hcy, the study results suggest that lowering Hcy, not increasing FA concentration, may be a 'primary' event that leads to improvement in renal function. Even more interestingly, although the baseline renal function, as well as tHcy, in the study subjects were normal, there was still the potential to increase GFR following intake of FA. It is unclear, however, whether improved renal function following FA administration can also be observed in older patients suffering from cardiovascular disease, in whom endothelial dysfunction is common, and if this effect can be beneficial in terms of major clinical outcomes, or neutral, as indicated by several clinical trials cited in the paper [1].

An additional effect of hyperhomocysteinaemia associated with cardiovascular risk and modulated by FA is post-translational protein $\mathrm{N} \varepsilon$-homocysteinylation, which may trigger autoimmune response and lead to the formation of potentially harmful specific antibodies, as shown in patients with early-onset coronary artery disease or renal insufficiency [3, 4]. Antibodies to $N \varepsilon$-homocysteinylated proteins may bind to them, with immune complex formation within the glomeruli and tubules; it is possible that lowering tHcy with FA may limit renal injury through mitigation of antibody formation. However, it seems unlikely that FA supplementation in a relatively short period of time would be sufficient to lower autoantibodies against $\mathrm{N} \varepsilon$-homocysteinylated proteins, despite a marked decrease in tHcy (as demonstrated in 2006) [5]. It is worth investigating whether improved renal function in young individuals following a three-month $\mathrm{FA}$ administration can affect $\mathrm{N} \varepsilon$-homocysteinylation and its potential consequences. Recently, elevated Hcy has been shown to specifically up-regulate the inflammatory process and oxidative stress that lead to podocyte injury in an animal model [6]. It could be speculated that attenuation of this mechanism contributes to increased GFR during FA administration, as reported by Mierzecki et al. [1]. Elucidation of the phenomenon of FA-induced improvement of renal function among healthy people is an attractive area of further research.

\section{Conflict of interest: none declared}

\section{References}

1. Mierzecki A, Makarewicz-Wujec M, Kłoda K et al. Influence of folic acid supplementation on coagulation, inflammatory, lipid, and the kidney function parameters in subjects with low and moderate content of folic acid in the diet. Kardiol Pol, 2015; 73: 280-286.

2. Wilk G, Osmenda G, Matusik P et al. Endothelial function assessment in atherosclerosis: comparison of brachial artery flow-mediated vasodilation and peripheral arterial tonometry. Pol Arch Med Wewn, 2013; 123: 443-452.

3. Kolarz M, Małyszko J, Stompór T et al. Antibodies against $\mathrm{N} \varepsilon$-homocysteinylated proteins in patients on different methods of renal replacement therapy. Clin Chem Lab Med, 2013; 51: 1093-1099.

4. Undas A, Jankowski M, Twardowska Met al. Antibodies to N-homocysteinylated albumin as a marker for early-onset coronary artery disease in men. Thromb Haemost, 2005; 93: 346-350.

5. Undas A, Stepien E, Glowacki R et al. Folic acid administration and antibodies against homocysteinylated proteins in subjects with hyperhomocysteinemia. Thromb Haemost, 2006; 96: 342-347.

6. Abais JM, Xia M, Li G et al. Nod-like receptor protein 3 (NLRP3) inflammasome activation and podocyte injury via thioredoxin-interacting protein (TXNIP) during hyperhomocysteinemia. J Biol Chem, 2014; 289: 27159-27168.

\section{Address for correspondence:}

Prof. Tomasz Stompór, MD, Department of Nephrology, Hypertension, and Internal Medicine, University of Warmia and Mazury, ul. Żołnierska 18, 10-561 Olsztyn, Poland, tel: +48 89 5386219, fax: +48 89 5337882, e-mail: stompin@mp.pl

Copyright (C) Polskie Towarzystwo Kardiologiczne 